\title{
Contextual stimuli and memory retrieval in pigeons
}

\author{
C. F. HICKIS, LEHA ROBLES, and DAVID R. THOMAS \\ University of Colorado, Boulder, Colorado 80309
}

\begin{abstract}
Pigeons were given free-operant successive discrimination training in which, on alternate days, two different interdimensional problems were employed (color positive and line angle negative; a different color negative and a different line angle positive). Between days, these problems could be construed as intradimensional ones. For one group, training was conducted on each problem in the presence of the same ambient (contextual) stimuli, while for a second group each problem was trained in a different context. For a third group, these two contexts were randomly related to the problems. Postdiscrimination stimulus generalization gradients showed that peak shifts were obtained in both the same context and the random context groups, but no peak shifts were found for the group which learned each problem in a different context. Results were consistent with Spear's (1973) treatment of animal memory which attributes retention test performance to the operation of a "context-elicited" retrieval process. An alternative uniprocess conditioning account of these and similar data was also discussed.
\end{abstract}

Spear $(1971,1973)$ has provided a theoretical account of animal memory which attributes much of forgetting to the lack of appropriate and/or a sufficient number of retrieval cues at the time of retention testing. According to this position, ambient (contextual) stimuli, present but inconsequential to the target learning task, can function as "reminders," eliciting retrieval of a memory concerning prior training if they are stored as attributes of that memory.'

Support for the argument that contextual stimuli can serve as retrieval cues was provided by Chiszar and Spear (1969). These investigators used two different $\mathrm{T}$-mazes located in two different rooms in a proactive interference paradigm with rats. One group was trained on a brightness discrimination in one of the mazes (Context 1 ), and a second group was trained to the same performance level in the other maze (Context 2). All subjects were then given reversal training of the original brightness discrimination in Context 1 . Results were that those subjects given original and reversal training in Context 1 made more errors and required more time to reach criterion on the reversal task than those subjects given original and reversal training in different contexts. Presumably, the presence of the same context

Preparation of this manuscript and the research reported herein was supported by NIMH Predoctoral Research Fellowship MH-05172 awarded to C. F. Hickis, and by NIH Research Grant HD-03486 and Training Grant M-10427 under the direction of D. R. Thomas. The authors wish to acknowledge the valuable comments of R. L. Jackson and S. F. Maier who read an earlier version of this manuscript. Requests for reprints should be sent to C. F. Hickis or D. R. Thomas, Department of Psychology, University of Colorado, Boulder, Colorado 80309. during original and reversal training elicited the memory of original training and this proactively interfered with performance on the reversal task. On the other hand, in the context change group, context functioned to delineate original and reversal training, thus protecting these subjects from proactive interference.

The idea that contextual cues can serve to delineate memory for particular training experiences was extended as part of a recent doctoral dissertation conducted in our laboratory (Miller, 1972). Chiszar and Spear (1969) had demonstrated indirectly that contextual cues facilitate retrieval of memory, i.e., retrieval of the Task 1 memory was inferred from the observed interference with acquisition of Task 2 performance. Miller's procedure permitted a direct assessment of the retrieval function by demonstrating that generalization test performance in a given context was consistent with the discrimination learning previously accomplished in that context.

Two of the eight groups actually run by Miller are important here. Pigeons were trained to discriminate a 576-nm light $(\mathrm{S}+)$ from a 555-nm light $(\mathrm{S}-)$. During this first stage of training, context conditions were HOUSELIGHT OFF + NOISE for all subjects. In the second stage, all subjects were trained on the reversal of the original discrimination task, i.e., $555 \mathrm{~nm}(\mathrm{~S}+)$ vs. $576 \mathrm{~nm}(\mathrm{~S}-)$. Groups 1 and 2 both received reversal training in a context different from that present during original learning (HOUSELIGHT ON + TONE). Finally, both groups were tested for stimulus generalization with a series of nine wavelengths symmetrically arranged around $555 \mathrm{~nm}$. Group 1 was tested in the same context in which reversal training took place (HOUSELIGHT ON + 
TONE), and Group 2 was tested in the same context in which original training took place (HOUSELIGHT OFF + NOISE). The result of intradimensional discrimination training (i.e., between two wavelengths) is typically a gradient with maximal responding displaced from $\mathrm{S}+$ so as to be farther removed from $\mathrm{S}$ (i.e., a peak shift, cf. Hanson, 1959; Purtle, 1973). In Miller's experiment, Group 1 showed a peak shift to $549 \mathrm{~nm}$, which is consistent with the reversal problem (555 $\mathrm{nm} \mathrm{S}+$ vs. $576 \mathrm{~nm} \mathrm{~S}-$ ) learned in the context in which testing took place. Group 2 also demonstrated a peak shift, responding maximally to $587 \mathrm{~nm}$, which is consistent with the discrimination problem learned first ( $576 \mathrm{~nm} \mathrm{~S}+$ vs. $555 \mathrm{~nm} \mathrm{~S}-$ ) and learned in the same context in which these subject were tested. Thus, although both groups received both original and reversal training and each problem was learned in a different context, the memory tests gave evidence of memory for only a single problem, i.e., that acquired in the context in which testing was conducted.

Now consider the following experiment reported by Lyons and Akins (1973). These investigators trained pigeons on two discrimination problems which alternated from day to day. On one day, the task was $555 \mathrm{~nm}(\mathrm{~S}+)$ vs. $15^{\circ}$ line $(\mathrm{S}-)$, and on alternate days, the task was $90^{\circ}$ line $(\mathrm{S}+)$ vs. $538 \mathrm{~nm}$ $(\mathrm{S}-)$. Training continued with these problems alternating on successive days until attainment of criterion on both discrimination tasks. Following mastery of the discrimination, all subjects were tested for generalization to stimuli falling along the wavelength and line-angle dimensions. The results were that all subjects demonstrated peak shifts along the wavelength dimension with maximal responding at a stimulus displaced from $555 \mathrm{~nm}$ so as to be farther from $538 \mathrm{~nm}$. No peak shifts were observed in the tests with line angle stimuli; however, the values of $90^{\circ}$ and $15^{\circ}$ are so widely separated that they do not normally lead to a peak shift in simple intradimensional training (Hearst, 1968). The Lyons and Akins finding is interesting because peak shifts were obtained along the wavelength dimension, even though discrimination training on any particular day was between two orthogonal stimuli. Such (interdimensional) training does not ordinarily produce peak shifts (Purtle, 1973). The fact that it did so in this case may be attributed to memory. The subjects did not separately encode and store the experiences of interdimensional problems on alternating days, thus both were retrieved during generalization testing. Functionally, it was as if they had learned a single problem involving both a wavelength discrimination and a line-angle discrimination.

Our analysis of the Miller experiment suggests that if a different context had been used on alternating days in the Lyons and Akins experiment, the memories would have been distinctively encoded and separately stored and thus would have been separately retrieved, depending upon the context present during testing. Under such conditions, no peak shift would be expected.

The present investigation was conducted in order to test this interpretation. In Experiment la, two groups of pigeons were compared. One of these was essentially a replication of the Lyons and Akins study; peak shifts were thus expected from this group. Unlike in their study, however, our line-angle stimuli were more closely spaced in the hope that this would result in peak shifts along both the line-angle and wavelength dimensions. In our second group, a different context differentiated odd from even days; we expected no peak shifts along either dimension from these subjects.

\section{EXPERIMENT 1a}

\section{Method}

Subjects. Twelve experimentally naive adult domestic pigeons obtained from a local supplier and maintained at $75 \%( \pm 5 \mathrm{~g})$ of their ad-lib body weights served as subjects. The birds were housed individually in a colony room on a 12 -h light/dark cycle and with continuous access to grit and water.

Apparatus. The experimental chambers were four identical sound-attenuating ice chests with interior dimensions of $31 \times 35$ $\times 36 \mathrm{~cm}$. Each chamber had a wire-mesh floor, and one wall constituted an "intelligence panel" constructed of aluminum with a 2.2 -cm-diam translucent plastic response key centered $20 \mathrm{~cm}$ above the floor. Approximately $15 \mathrm{~cm}$ directly below the key, a $6.3-\mathrm{cm}$ square aperture allowed access to mixed grain from a solenoid-operated hopper. Behind the front wall directly to the right of the aperture was a $10-\mathrm{cm}$-diam speaker which delivered 80-dB (SPL) white noise inside the chamber (GrasonStadler Model $901 \mathrm{~B}$ white-noise generator). A fan mounted behind the front wall provided ventilation as well as additional masking noise.

Stimuli were projected onto the keys by Industrial Electronic Engineers in-line display cells (No. 10-01-3040-1815) mounted $.64 \mathrm{~cm}$ behind the keys. Stimuli of nominal peak transmitted wavelengths $501,538,555,576$, and $606 \mathrm{~nm}$ were produced by Kodak Wratten filters $65,74,99,73$, and $72 \mathrm{~B}$, respectively. White lines with angular orientations of $60^{\circ}, 75^{\circ}, 90^{\circ}$ (vertical), $105^{\circ}$, and $120^{\circ}$ were $3 \mathrm{~mm}$ wide $\times 21 \mathrm{~mm}$ long and bisected the otherwise dark keys. The filters were transilluminated by individual G.E. miniature lamps (No. 1815) drawing $200 \mathrm{~mA}$ current. While no attempt was made to control for luminosity differences which do occur with these projectors, Lyons and Klipec (1971) have shown that pigeons respond systematically to wavelength differences and not intensity differences in a stimulus generalization test involving chromatic stimuli of the values employed here. Houselights could be provided by illuminating a single G.E. No. 1829 lamp located behind a $3 \times 31 \mathrm{~cm}$ strip of diffusing white Plexiglas attached across the entire top of the intelligence panel. The only other source of illumination in the chambers was provided by an ESB 12-V lamp situated above the food hopper and illuminated for the entire duration of each hopper presentation. Programming of scheduled events was accomplished by standard relay circuitry located in an adjoining control room.

Procedure. On the first day, all birds were magazine-trained, hand-shaped to peck the key, and allowed to earn 50 reinforcers delivered on a continuous reinforcement schedule. During the next 3 days, the mean interreinforcement interval was gradually lengthened until a variable-interval 1-min (VI 1-min) schedule was in effect. During initial training, context conditions were HOUSELIGHT OFF + NOISE and the key was continuously illuminated for half of the subjects by $555 \mathrm{~nm}$ and for the other half by a $90^{\circ}$ 
(vertical) line. The reinforcer throughout this experiment was $3 \mathrm{sec}$ of access to the hopper of mixed grain. Four additional days of VI 1-min single-stimulus training to $555 \mathrm{~nm}$ or $90^{\circ}$ line for 30 min each day was then followed by nondifferential training.

Nondifferential training. Pilot work had indicated that the transition from single stimulus to compound discrimination training proceeds with far less distuption in responding if a number of days of nondifferential training precedes the onset of discrimination training. The 12 subjects were systematically divided into two groups ( $n=6$ in each) so as to closely match response rates during single-stimulus training. For Group 1, training proceeded as follows: Daily sessions were divided into 30 successive components each of 1-min duration with no intervening blackouts. On odd-numbered days (Days 1, 3, 5, 7, and 9), responding in the presence of $555 \mathrm{~nm}$ and $75^{\circ}$ line were reinforced according to the same VI 1-min schedule used during single-stimulus training. On even-numbered days (Days 2, 4, 6, 8 , and 10), responding was reinforced likewise, but the stimuli were $90^{\circ}$ line and $576 \mathrm{~nm}$. Thus each subject was exposed to two different interdimensional "problems" which alternated from day to day. Stimuli within a session were each presented successively for 1-min component durations in a quasirandom series, with the stipulation that no more than three consecutive periods with the same stimulus could occur. Context conditions were HOUSELIGHT OFF + NOISE at all times. Subjects in Group 2 were treated identically except for the context in which alternating daily problems were conducted. On odd-numbered days, the stimuli associated with reinforcement were $555 \mathrm{~nm}$ and $75^{\circ}$ line and the context was HOUSELIGHT OFF + NOISE. Note that these conditions are identical to those for Group 1. On evennumbered days, the stimuli were, as for Group $1,90^{\circ}$ line and $576 \mathrm{~nm}$, but the context was different, i.e., HOUSELIGHT ON + TONE $[1,000 \mathrm{~Hz}$ at $85 \mathrm{~dB}$ (SPL)]. Training proceeded as described earlier, with Group 1 having alternating problems in the same context, while Group 2 had the same alternating problems but with each in a different context. Nondifferential training was conducted for a total of 10 days, that is, 5 days on each problem, and was immediately followed on Day 11 by discrimination training.

Discrimination training. Stimulus conditions and context conditions were the same during discrimination training as they were in nondifferential training. However, the subjects were now trained on odd days with the problem $555 \mathrm{~nm}(\mathrm{~S}+)$ vs. $75^{\circ}$ line $(\mathrm{S}-)$ and on even days with the problem $90^{\circ}$ line $(\mathrm{S}+$ ) vs. $576 \mathrm{~nm}$ $(\mathrm{S}-)$. This was accomplished by reinforcing responses in the presence of $\mathrm{S}+\mathrm{s}$ according to the same VI 1-min schedule employed earlier, and extinguishing responses in the presence of $\mathrm{S}-\mathrm{s}$. Reinforced and nonreinforced components were of $1-\mathrm{min}$ duration, and daily sessions comprised a random ordering of $15 \mathrm{~S}+$ and $15 \mathrm{~S}$ - presentations. No intervening blackouts separated successive components and no more than three consecutive $S+$ or S-components could occur. A 3-sec changeover delay in which a reinforcer could not be made available during the first $3 \mathrm{sec}$ of an $\mathbf{S}+$ component was employed in order to reduce the possibility of $\mathrm{S}+$ onset acting as a conditioned reinforcer maintaining responding at the end of an S - component. Subjects in Group 1 were run on the alternating discrimination problems each in the HOUSELIGHT OFF + NOISE context, while Group 2 received the alternating problems with context conditions being HOUSELIGHT OFF + NOISE on odd days and HOUSELIGHT ON + TONE on even days. Subjects were run with daily problem alternation until attainment of a criterion of two consecutive sessions on the same problem with at least $85 \%$ of all responses made in the presence of $\mathrm{S}+$. Following attainment of criterion, subjects were tested for stimulus generalization as subsequently discussed.

Subjects were randomly assigned to testing conditions prior to their completion of discrimination training. Three subjects in each group were tested with stimuli from the wavelength dimension and three with line-angle stimuli. Those subjects to be tested with wavelengths were run with daily problem alternations until attainment of criterion on the $555 \mathrm{~nm}$ vs. $75^{\circ}$ line problem and then were tested on the next day on which that problem was scheduled. Those subjects to be tested with line angle were run with daily problem alternations until attainment of criterion on the $90^{\circ}$ line vs. $576 \mathrm{~nm}$ problem and then were tested on the next day on which this problem was scheduled. Note that it was not required that subjects meet criterion on both problems before testing; rather, they were run to criterion on the relevant problem, i.e., the problem in which $S+$ occurred along the to-be-tested dimension.

Generalization testing. Following attainment of criterion on the discrimination task, all subjects were tested for stimulus generalization. Following a 10 -min reinforced warm-up on the appropriate discrimination, a series of blocks of five wavelengths $(501,538,555,576$, and $606 \mathrm{~nm})$ or five line angles $\left(60^{\circ}, 75^{\circ}\right.$, $90^{\circ}, 105^{\circ}$, and $120^{\circ}$ ) was successively presented, with no responses being reinforced and no intervening blackouts. The stimuli remained on the key for 30-sec each, and each block consisted of a different random ordering of each of the five stimuli. The test was ended for all subjects after 10 blocks had been presented. Group 1 was tested in the same context used throughout trainingHOUSELIGHT OFF + NOISE. Group 2 was tested in the same context in which the relevant discrimination problem was learned, that is, HOUSELIGHT OFF + NOISE for subjects tested with wavelengths and HOUSELIGHT ON + TONE for those tested with line angles.

\section{Results}

All subjects performed comparably during magazine training, response acquisition, and reinforcement density reduction to VI $1 \mathrm{~min}$. Following the initial 4 days of VI 1-min single-stimulus training, all subjects were responding consistently and were assigned to groups so that Group $1 \mathrm{had}$ an average response rate (corrected for total hopper time) of 49 responses/min, with a range from 29 to 71 responses/min, and Group 2 had an average response rate of 53 responses/min, ranging from 27 to 88 responses/min. The performance of these groups did not differ statistically $(U=17, p>.90$, two-tailed $)$. There was also no difference in response rates between subjects having the 555-nm stimulus and those having the $90^{\circ}$ stimulus $(U=12.5, \mathrm{p}>.40)$.

During the 10 days of nondifferential training, all subjects responded consistently to all four stimuli. Discrimination ratios, of the form (responses to Stimulus 1)/(responses to Stimulus $1+$ responses to Stimulus 2), computed on each problem were the basis for comparison. Group 1's discrimination ratio averaged over the last 2 days on the $555 \mathrm{~nm}-75^{\circ}$ line problem was .51 (range from .44 to .55); Group 2's ratio on this problem averaged .54 (range from .46 to .58). On the $90^{\circ}$-line/576-nm problem, discrimination ratios averaged .52 (range from .46 to .57 ) for Group 1 and .50 (range .43 to .54 ) for Group 2. None of these differences among these ratios approached statistical reliability (all Us $>12.5$, ps $>$ .40 ). Thus, both groups were responding nondifferentially and did not differ at the outset of discrimination training.

Acquisition of discriminative performance pro- 
ceeded gradually, with most subjects continuing to respond nondifferentially for the first 10 days of discrimination training. After this, response rates to $\mathrm{S}-\mathrm{s}$ began to decrease while responding was maintained in the presence of $S+s$. The average numbers of days to criterion on the relevant problems were 44 days (range from 28 to 62) for Group 1 and 20 days (range from 19 to 42 ) for Group 2, a difference which was significant $(U=4, p<.03)$.

Discrimination ratios $(\mathrm{S}+$ responses/total responses to $\mathrm{S}+$ and $\mathrm{S}-$ ) on both discrimination tasks for Groups 1 and 2 are given in Table 1. Column 1 gives discrimination ratios for individual subjects on the relevant discrimination problem, and Column 2 gives discrimination ratios on the irrelevant problem averaged across the last 2 days of training on each problem. Note that the majority of subjects were discriminating quite well and exceeded criterion on each problem immediately prior to generalization testing. Average discrimination ratios for Groups 1 and 2 on the relevant discrimination problem were .88 and .87 , respectively, and discrimination ratios on the irrelevant problem were .86 and .83 , respectively. None of the statistical comparisons among these scores approached significance (all Us $>13$, ps $>.48$ ). Note, however, that one subject in Group 1 (L25) had a much lower discrimination ratio on the irrelevant discrimination task (.73) than on the relevant one, as did two subjects in Group 2 (L35 and L16) with discrimination ratios of .77 and .73, respectively.

The data of primary interest, of course, are the results of generalization testing. Figures 1 and 2 display individual subjects' generalization gradients plotted as the total number of responses emitted to each of the test stimuli. The upper three panels in each of the figures show the results for those sub-

Table 1

Discrimination Ratios Averaged Across the Last 2 Days of Discrimination Training in Experiment 1a

\begin{tabular}{lcccccc}
\hline $\begin{array}{c}\text { Subject } \\
\text { Number }\end{array}$ & \multicolumn{7}{c}{ Relevant Task } & \multicolumn{7}{c}{ Irrelevant Task } \\
\hline \multicolumn{7}{c}{ Group 1 } \\
L33 & $90^{\circ}$ & 576 & .86 & 555 & $75^{\circ}$ & .93 \\
L31 & $90^{\circ}$ & 576 & .89 & 555 & $75^{\circ}$ & .88 \\
L25 & $90^{\circ}$ & 576 & .85 & 555 & $75^{\circ}$ & .73 \\
L20 & 555 & $75^{\circ}$ & .87 & $90^{\circ}$ & 576 & .90 \\
L5 & 555 & $75^{\circ}$ & .90 & $90^{\circ}$ & 576 & .89 \\
L17 & 555 & $75^{\circ}$ & .91 & $90^{\circ}$ & 576 & .85 \\
Mean & & & .88 & & & .86 \\
& & & \multicolumn{6}{c}{ Group 2 } & & \\
L14 & $90^{\circ}$ & 576 & .88 & 555 & $75^{\circ}$ & .86 \\
L35 & $90^{\circ}$ & 576 & .87 & 555 & $75^{\circ}$ & .77 \\
L12 & $90^{\circ}$ & 576 & .89 & 555 & $75^{\circ}$ & .87 \\
L16 & 555 & $75^{\circ}$ & .85 & $90^{\circ}$ & 576 & .73 \\
L22 & 555 & $75^{\circ}$ & .90 & $90^{\circ}$ & 576 & .89 \\
L18 & 555 & $75^{\circ}$ & .86 & $90^{\circ}$ & 576 & .89 \\
Mean & & & .87 & & & .83 \\
\hline
\end{tabular}

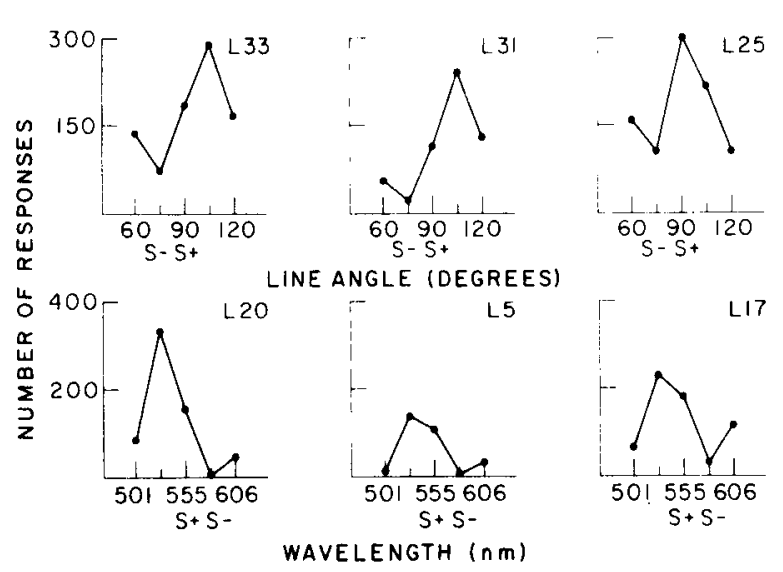

Figure 1. Individual subjects' generalization gradients for Group 1 in Experiment 1a. See text for explanation.

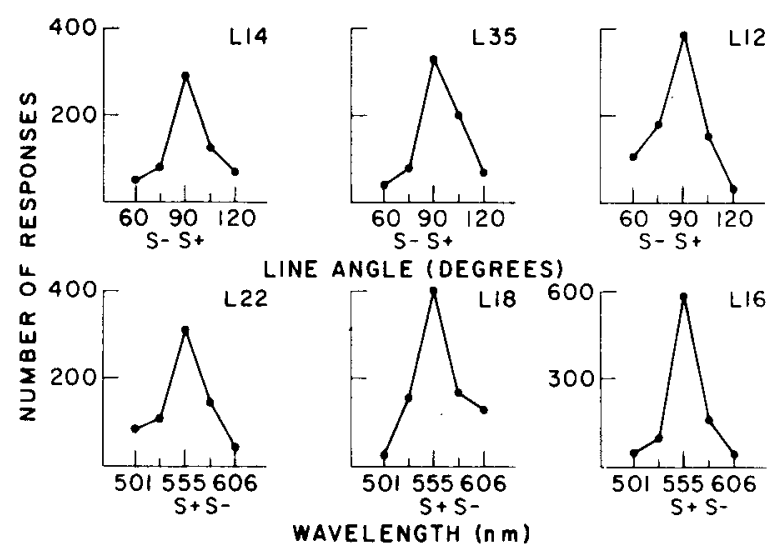

Figure 2. Individual subjects' generalization gradients for Group 2 in Experiment 1a. See text for explanation.

jects tested with line-angle stimuli and the lower three panels present the results for subjects tested with wavelength stimuli. For convenience in identifying the prior training stimuli, these values have been labeled $\mathrm{S}+$ for $90^{\circ}$ line and $555 \mathrm{~nm}$ and $\mathrm{S}-$ for $75^{\circ}$ line and $576 \mathrm{~nm}$. Remember, however, that the $\mathrm{S}+$ and $S$ - cues within the same dimension were actually seen on alternate days during the course of training. As shown in Figure 1, five of the six subjects in Group 1 demonstrated a peak shift, with only Subject L25, tested with line angles, failing to do so. This finding contrasts with Group 2's generalization test performance, shown in Figure 2. Note that in Group 2 all six subjects responded maximally to $\mathrm{S}+$, i.e., no peak shifts were observed. One further difference to note between the generalization test performance of Group 1 and Group 2 is the number of responses emitted in the presence of the $S$ - cues. Notice that in Group 1 the minimal number of responses to any test stimulus was in each case located at $\mathrm{S}$-. This contrasts with the performance of Group 2, in which case responding dropped off gradually from $\mathrm{S}+$ with the minimal number of responses located not at $S-$, but at stimulus values most distant from $\mathrm{S}+$. 


\section{Discussion}

The results of Group 1 confirm the prediction made earlier that peak shifts would occur in the postdiscrimination generalization gradients of subjects trained on alternate days with two different interdimensional discrimination problems, which can be viewed across days as intradimensional problems. Five of six subjects in this group demonstrated a peak shift when tested.

Recall that all subjects except L25 were discriminating with ratios greater than .85 on the irrelevant task immediately prior to generalization testing. Subject L25 had a discrimination ratio of only .73 at this time. It has been shown that the best predictor of peak shift is a high overall discrimination ratio following training (Wheatley \& Thomas, 1974). It seems likely that had $\mathrm{L} 25$ received additional training sufficient to bring its discrimination ratio into line with that of the other subjects in Group 1, it too would have shown a peak shift.

Consistent with the finding of peak shifts in five of six subjects in Group 1 is the finding in all six cases of lower responding to the $S-$ value than to a value still farther removed from $\mathrm{S}+$. It is typical for generalization gradients following simple intradimensional discrimination training to show a trough in the vicinity of S - (Hearst, 1969; Purtle, 1973).

Furthermore, as predicted, no peak shifts were found in Group 2, for which the two alternative problems were trained each in a different context. Note also that in no case was the minimum of responding at the " $\mathrm{S}$-" value. This finding, along with the absence of peak shifts in this group, is compelling evidence suggesting that, for these subjects, the functional S - was an orthogonal stimulus and not the value labeled as $\mathrm{S}$ - in the figure.

One additional point merits further discussion. It was found that Group 1 took longer to achieve criterion on the relevant discrimination problem than did Group 2. This finding is consistent with our interpretation that, in Group 2, the subjects treated the task as involving two distinct interdimensional problems with no generalization between what was learned on odd days and what was learned on even days. In Group 1, however, the presence of peak shifts reveals that the subjects construed the task as involving two intradimensional problems as well as the interdimensional ones programmed by the experimenters. Gradient interaction theory (Spence, 1937), which predicts the peak shift, also predicts more difficulty in acquiring an intradimensional than in acquiring an interdimensional discrimination because of the generalization of excitatory and inhibitory tendencies which occurs in the former case and not in the latter one. The smaller amount of training received by Group 2 cannot account for the lack of peak shifts in this group. Thomas (1962) showed that in simple intradimensional training peak shifts are evident quite early in training, although they increase in magnitude as training is continued. There is even some evidence to suggest (cf., Terrace, 1966) that very extensive training may eliminate peak shifts which had been present earlier in training. Finally, as mentioned earlier, Wheatley and Thomas (1974) have shown that the discrimination ratio is the best predictor of peak shift, and the discrimination ratios were comparable in Groups 1 and 2.

\section{EXPERIMENT 1b}

There is one obvious procedural difference between Groups 1 and 2 in Experiment 1a which must be considered before any firm conclusions may be drawn. Our argument is that the presence of differential contextual stimuli in Group 2 served to isolate memory for the training tasks, and thus, during the generalization test, these contextual cues acted to retrieve the appropriate information specifying interrather than intradimensional training, that is, no peak shifts were found. For Group 1, however, since both problems were experienced in the same context, these subjects stored both inter- and intradimensional information. Then, during the generalization test, the context served to retrieve the memory of both inter-and intradimensional training tasks, and peak shifts were thus obtained. Presumably the absence of peak shifts in Group 2 occurred by virtue of the unique correlation which the different contexts had with each problem for these subjects. Yet, in comparing Groups 1 and 2, it might be argued that the mere presence of differential contextual stimuli in Group 2 was sufficient to eliminate peak shifts in that group.

Experiment $1 \mathrm{~b}$ was conducted in order to assess this possibility. Six pigeons were trained in the same fashion as Groups 1 and 2 in Experiment $1 \mathrm{a}$, and then were tested for stimulus generalization in an identical manner. Additionally, the same contextual stimuli used with Group 2 were also present during training here. However, now the different contexts were randomly alternated. That is, there was no systematic correlation between discrimination problems and contextual stimuli. If we are correct in our assumption that the context conditions for Group 2 of Experiment la served to isolate memory for the discrimination task problems, then we would expect to find peak shifts in Experiment $1 \mathrm{~b}$, because here the context no longer acts in this fashion.

\section{Method}

Subjects. Six experimentally naive domestic pigeons, obtained from a local supplier and maintained as in Experiment 1a, served as subjects.

Apparatus. The operant chambers and associated relay cir cuitry used in Experiment la were also used here.

Procedure. Magazine training, response acquisition, reinforcement density reduction, and single-stimulus training were conducted in a fashion identical to that in Experiment 1a. Half 
of the subjects were trained with the key illuminated by $555 \mathrm{~nm}$ and the other half with $90^{\circ}$ line. Following 4 days of single stimulus training, all subjects were given 10 days of nondifferential training, with all four stimuli being equally reinforced according to the same VI 1-min schedule used earlier. On odd-numbered days, the stimuli were $555 \mathrm{~nm}$ and $75^{\circ}$ line, and on even-numbered days, the stimuli were $90^{\circ}$ line and $576 \mathrm{~nm}$. During nondifferential and subsequent discrimination training, two alternative contexts (HOUSELIGHT OFF + NOISE and HOUSELIGHT $\mathrm{ON}+\mathrm{TONE}$ ) were present, but instead of alternating from day to day correlated with a particular interdimensional task as in Experiment 1a, in Experiment lb context conditions were arranged to occur in a random sequence. On some days, the HOUSELIGHT OFF + NOISE context accompanied the $555-\mathrm{nm} / 75^{\circ}$-line problem, and on some days, that context was associated with the $90^{\circ}$-line/576-nm problem. The same held true for the HOUSELIGHT ON + TONE context. Context conditions on any particular day were determined as follows. Each block of 10 days was divided into 5 days with each context. A table of random numbers determined which context would be given on a particular day. The only restriction on "true" randomness was that no more than 3 consecutive days with the same context could occur. Although context conditions were randomly related to problems, training proceeded with each problem strictly alternating from day to day. Ten days of nondifferential training were immediately followed by discrimination training.

Discrimination training proceeded as described for Experiment 1a. On odd-numbered days, responses to $555 \mathrm{~nm}$ were reinforced according to a VI 1-min schedule and responses to $75^{\circ}$ line were extinguished. On even-numbered days, responses to $90^{\circ}$ line were reinforced and responses to $576 \mathrm{~nm}$ were extinguished. As in Experiment 1a (Group 2), two alternative contexts were provided here, but now these contextual stimuli were randomly related to each problem. Context conditions on any particular day were determined as described for nondifferential training. Each block of 10 days consisted of a random ordering of 5 days with each of the different contexts. As in Experiment la, the subjects were run on the alternating discrimination problems until attainment of criterion of two consecutive daily sessions with at least $85 \%$ of all responses in the presence of the $S+$ cue from the to-betested dimension.

Following attainment of criterion on the discrimination task, all subjects were tested for stimulus generalization in extinction. Half of the subjects were tested with line-angle stimuli and half were tested with wavelength stimuli. Since context conditions were randomly related to discrimination task conditions, it was arbitrarily decided to test for line-angle generalization in the HOUSELIGHT OFF + NOISE context and to test for wavelength generalization in the HOUSELIGHT ON + TONE context. The remaining procedural details for generalization testing were identical to those for Experiment la.

\section{Results and Discussion}

All subjects performed comparably during magazine training, response acquisition, and reinforcement density reduction. Following 4 days of singlestimulus training, response rates averaged 55 responses/ $\mathrm{min}$, with a range from 22 to 68 responses/min. Following 10 days of nondifferential training, all subjects were responding with essentially equal rates to the cues from each problem with an average discrimination ratio of .53 (range from .45 to .54 ) on the $555 \mathrm{~nm}-75^{\circ}$ line problem and .51 (range from .46 to .55 ) on the $90^{\circ}$ line- $576 \mathrm{~nm}$ problem. For statistical purposes, the group in Experiment $1 \mathrm{~b}$ was compared with Groups 1 and 2 from Experiment 1a.
A Kruskal-Wallace analysis of variance indicated that the three groups were not reliably different from each other during single-stimulus training $(\mathrm{H}=2.23$, $\mathrm{df}=2, \mathrm{p}>.30$, two-tailed) or on discrimination ratios averaged across the last 2 days of nondifferential training on each problem ( $\mathrm{Hs}<1.0$ for both comparisons).

Acquisition of discriminative performance proceeded more slowly for subjects in Experiment $1 \mathrm{~b}$ than for the two groups in Experiment 1a. It took these subjects an average of 50 days (range from 34 to 71$)$ to reach criterion on the relevant discrimination task as compared to 44 days for Group 1 and 29 days for Group 2 in Experiment 1a. These groups were reliably different from each other $(H=6.35$, $\mathrm{df}=2, \mathrm{p}<.05$ ), and subsequent analyses revealed that the group in Experiment $1 \mathrm{~b}$ was reliably different from Group 2 in Experiment 1a $(U=3, p<.02)$, but the difference between this group and Group 1 was not significant $(\mathrm{U}=9, \mathrm{p}=.18)$.

Table 2 gives individual subjects' discrimination ratios on each problem immediately prior to the generalization test. Column 1 shows discrimination ratios on the relevant problem and Column 2 shows discrimination ratios on the irrelevant problem. Notice that the ratios reported here are comparable to those from Experiment la given in Table 1. All subjects were discriminating well, with ratios greater than .85 on the relevant problem immediately prior to generalization testing. As inspection of Column 2 reveals, the majority of subjects in this experiment also reached criterion on the irrelvant problem prior to testing. Note, however, that two of the subjects in this group (L11 and L19) were not discriminating as well on the irrelevant task, with ratios of .80 and .71, respectively.

Figure 3 displays individual subjects' generalization gradients plotted as the total number of responses emitted during the test to each of the stimuli. The upper panels present subjects tested with line-angle stimuli in the HOUSELIGHT OFF + NOISE context and the lower panels contain subjects tested with wavelength stimuli in the HOUSELIGHT ON + TONE context. Notice that four of six subjects in this group responded maximally to stimulus values of

Table 2

Discrimination Ratios Averaged Across the Last 2 Days of Discrimination Training in Experiment 16

\begin{tabular}{lccccccc}
\hline $\begin{array}{l}\text { Subject } \\
\text { Number }\end{array}$ & \multicolumn{3}{c}{ Relevant Task } & \multicolumn{3}{c}{ Irrelevant Task } \\
\hline L11 & $90^{\circ}$ & 576 & .87 & 555 & $75^{\circ}$ & .80 \\
L3 & $90^{\circ}$ & 576 & .90 & 555 & $75^{\circ}$ & .92 \\
L7 & $90^{\circ}$ & 576 & .88 & 555 & $75^{\circ}$ & .89 \\
L19 & 555 & $75^{\circ}$ & .92 & $90^{\circ}$ & 576 & .71 \\
L27 & 555 & $75^{\circ}$ & .86 & $90^{\circ}$ & 576 & .89 \\
L5 & 555 & $75^{\circ}$ & .89 & $90^{\circ}$ & 576 & .89 \\
Mean & & & .89 & & & .85 \\
\hline
\end{tabular}




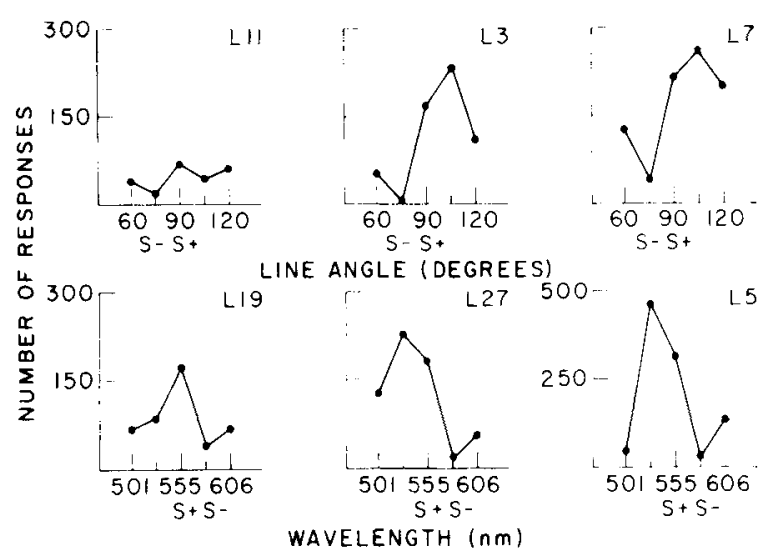

Figure 3. Individual subjects' generalization gradients in Experiment $1 b$. See text for explanation.

$105^{\circ}$ line and $538 \mathrm{~nm}$, i.e., a peak shift was obtained. Two subjects, L11, tested with lines, and L19, tested with wavelengths, did not show peak shifts, responding maximally to the prior training stimulus. Responding of Subject L11 was at a low rate and quite erratic during the course of testing, and it is questionable as to whether a reliable gradient was obtained. Subject L11 did not respond at all on $41 \%$ of the test stimulus presentations and then responded to only two or three of the stimuli within a block, whereas all remaining subjects in this and the prior experiment responded consistently throughout the test. Note further that both Subjects L11 and L19 were not discriminating well on the irrelevant task prior to generalization testing (discrimination ratios $=.80$ and .71 , respectively). This finding is like that observed with Subject L25 from Group 1 in Experiment 1a. That is, these three subjects did not achieve as high an overall level of discriminative performance as did all of the remaining subjects in both experiments. These three subjects were also the only ones who failed to exhibit peak shifts, whereas the remaining subjects in their groups did. Again, it seems reasonable to speculate that if $\mathrm{L} 11$ and $\mathrm{L} 19$ had received additional training so as to improve their discrimination performance and/or to make their generalization test performance more reliable, they too would have revealed peak shifts in their generalization gradients.

Note that, as with Group 1 in Experiment 1a, all subjects in Experiment $1 \mathrm{~b}$ exhibited a minimum of responding at the $\mathrm{S}-$ value. This did not happen even once with Group 2.

\section{GENERAL DISCUSSION}

The results of these experiments clearly suggest that contextual stimuli can serve as effective retrieval cues functioning to recall memory concerning training if they are stored as attributes of that memory. In Experiment 1a, it was shown that the presence of differential contextual stimuli associated with each of two interdimensional discrimination tasks served to isolate memory for these tasks, whereas the absence of such differential cues allowed the memories to interact. That interaction was reflected in three ways: (1) The discrimination was more difficult for subjects in Group 1 than for subjects in Group 2, presumably because of the generalization of excitatory and inhibitory tendencies within each dimension. (2) In generalization testing, peak shifts were observed in Group 1 but not in Group 2, again suggesting that the training was functionally (in part) intradimensional. (3) A trough of responding with a minimum at "S - " was observed in Group 1 but not in Group 2, further demonstrating the intradimensional aspect of what had been learned. Finally, in Experiment $1 \mathrm{~b}$, it was shown that the context served this function by virtue of its unique correlation with the reinforcement contingencies present in each problem rather than being due to the mere presence of these cues during training. A group which experienced both contexts uncorrelated with the two interdimensional problems performed similarly to Group 1 in Experiment 1a, which had experienced both problems in the same context. Thus our findings are consistent with those of Chiszar and Spear (1969) and Miller (1972) in suggesting that contextual stimuli can indeed serve as retrieval cues, eliciting a memory concerning prior training when they have previously been stored as attributes of that memory.

There is, however, an alternative theoretical account of these and similar data that should be considered. Recall that the test we used and those used by Chiszar and Spear and by Miller assessed contextual effects in a context in which some aspect of prior training took place. In all of these experiments, when tested in a particular context, subjects behaved in a manner consistent with what was learned in that context. There are available models of associative learning which consider the entire learning situation as an aggregate of potentially conditionable stimuli (e.g., Mackintosh, 1975; Rescorla \& Wagner, 1972). Such views maintain that contextual stimuli as well as explicitly programmed stimuli are conditioned as part of the stimulus configuration present during training. Then, during testing, subjects behave in the presence of elements from this configuration as they would have if the entire configuration had been present. Accordingly, when each problem was trained in a different context (Group 2, Experiment 1a), two unique stimulus configurations were formed [(Context $1+555 \mathrm{~nm}$ vs. $75^{\circ}$ line) and (Context $2+90^{\circ}$ line vs. $576 \mathrm{~nm}$ )]. During the test conducted in Context 1 , since it was part of the configuration containing $555 \mathrm{~nm}$ vs. $75^{\circ}$ line, subjects behaved in a manner consistent with that interdimensional 
problem, i.e., no peak shifts were found. The same holds true for subjects tested in Context 2. For Group 1, however, since the same context was present for both problems, only one stimulus configuration was formed (Context $+555 \mathrm{~nm}$ vs. $75^{\circ}$ line $+90^{\circ}$ line vs. $576 \mathrm{~nm}$ ). Note that this configuration contains both inter- and intradimensional information. Thus, peak shifts would be expected from these subjects.

Such an account of the present data makes it unnecessary to invoke a memory construct as an explanatory mechanism. In fact, it makes suspect any conclusions concerning memory in which retention testing is conducted in the presence of some aspect of the stimulus configuration present during training. There are, however, implications of a memorial interpretation which may provide a test of these two alternative explanations. For example, if the memory interpretation is correct, then the context might be used to briefly remind subjects of particular training contingencies. Subsequently, in the absence of those contextual stimuli, these subjects should behave as if the training contingencies appropriate to that context were still present. According to a strict conditioning model, however, such a manipulation should have no effect. Research is currently underway in our laboratory exploring the effectiveness of the context as a brief "reminder" cue.

\section{REFERENCES}

Chiszar, D. A., \& Spear, N. E. Stimulus change, reversal learning and retention in the rat. Journal of Comparative and Physiological Psychology, 1969, 69, 190-195.

Hanson, H. M. Effects of discrimination training on stimulus generalization. Journal of Experimental Psychology, 1959, 58, 321-334.

HEARST, E. Discrimination learning as the summation of excitation and inhibition. Science, 1968, 196, 1303-1306.

HEARST, E. Excitation, inhibition, and discrimination learning. In N. J. Mackintosh \& W. K. Honig (Eds.), Fundamental issues in associative leaming. Halifax: Dalhousie University Press, 1969.

Lyons, J., \& Akins, F. The effect of two alternating interdimensional discrimination tasks on stimulus control. Bulletin of the Psychonomic Society, 1973, 2, 323. (Abstract)
Lyons, J., \& KLIPEC, W. D. Color mixing with Kodak Wratten filters. Journal of the Experimental Analysis of Behavior, 1971, $15,232$.

Mackintosh, N. J. A theory of attention: Variations in associability of stimuli with reinforcement. Psychological Review, 1975, 82. 276-298.

MILLER, J. T. The effect of contextual cue change during reversal training on postdiscrimination generalization gradients. Doctoral dissertation, University of Colorado, 1972.

Purtle, R. Peak shift: A review. Psychological Bulletin, 1973, 80, 408-421.

Rescorla, R. A., \& WAGner, A. R. A theory of Pavlovian conditioning: Variations in the effectiveness of reinforcement and nonreinforcement. In A. H. Black \& W. F. Prokasy (Eds.), Classical conditioning II: Current theory and research. New York: Appleton-Century-Crofts, 1972.

SPEAR, N. E. Forgetting as retrieval failure. In W. K. Honig \& P. James (Eds.), Animal memory. New York: Academic Press, 1971.

SPEAR, N. E. Retrieval of memory in animals. Psychological Review, 1973, 80, 163-194.

Spence, K. W. The differential response in animals to stimuli varying within a single dimension. Psychological Review, 1937. 44, 430-444.

Terrace, H. S. Stimulus control, In W. K. Honig (Ed.), Operant behavior: Areas of research and application. New York: Appleton-Century-Crofts, 1966.

Thомаs, D. R. The effects of drive and discrimination training on stimulus generalization. Journal of Experimental Psychology, $1962,64,24-28$.

Whe atley, K. L., \& Thomas, D. R. Relative and absolute density of reinforcement as factors influencing the peak shift. Journal of the Experimental Analysis of Behavior, 1974, 22. 409-418.

\section{NOTE}

1. It might be appropriate at this point to clarify our use of certain terms in the manuscript. The word "memory" refers to an internal representation of a prior training episode. By "contextual stimuli," we mean those always present, ambient stimuli which are part of the chamber in which conditioning takes place. This includes houselights, noise, tones, floor and wall texture, etc. Contextual stimuli are relatively inconsequential to the target learning task, but are merely present at the time training takes place: the terms "contextual stimuli," "contextual cues," and "context" are used interchangeably.

(Received for publication September 16, 1976; revision accepted December $23,1976$. 